\title{
Design of INS data acquisition device based on STM32
}

\section{Dong He, * Zhenrong Zhang, Feng Yang}

School of Computer, Electronics and Information, Guangxi University, Nanning 530004, Guangxi, China

*zzr76@gxu.edu.cn(Corresponding author)

Keywords: Inertial navigation system, data acquisition device, A/D conversion, kalman filtering Abstract. We design a new data acquisition device based on STM32 for Inertial navigation system. The data acquisition device has the character of simple structure, low power consumption, miniaturization. And the experiments show that this data acquisition device has the function of the A/D conversion and kalman filtering.

\section{Introduction}

Inertial navigation system (INS) is an autonomous navigation system, which is not dependent on external information, nor transmit information to the outside[1]. Therefore, because of its good concealment, inertial navigation system is widely used in space exploration, space navigation, deep-sea extreme environment navigation and land navigation, etc.

The basic operating principle of the inertial navigation system is that uses a computer, motion sensors (accelerometer) and rotation sensors (gyroscope) to continuously calculate via dead reckoning the position, orientation, and velocity (direction and speed of movement) of a moving object without the need for external references[2].In fact, the computer can not directly use the measured value of the gyroscope and accelerometer for navigation calculations. here's why:

(a).The measured value of the gyroscope and accelerometer is analog signal. The navigation computer doesn't recognize analog signal, in order to enable it to work, we need to convert analog signal to digital signal (A/D conversion).

(b). The measured value of the gyroscope and accelerometer contains error of measurement. These error is mainly by the gyro drift and accelerometer zero bias and error of initial conditions. The error of inertial navigation system mainly by the gyro drift and accelerometer zero bias and error caused by the initial conditions. As mentioned above, the error of inertial navigation system has three kinds of periodic oscillation (schuler cycle, foucault cycle and earth's rotation cycle) and random error of gyro drift growth over time.

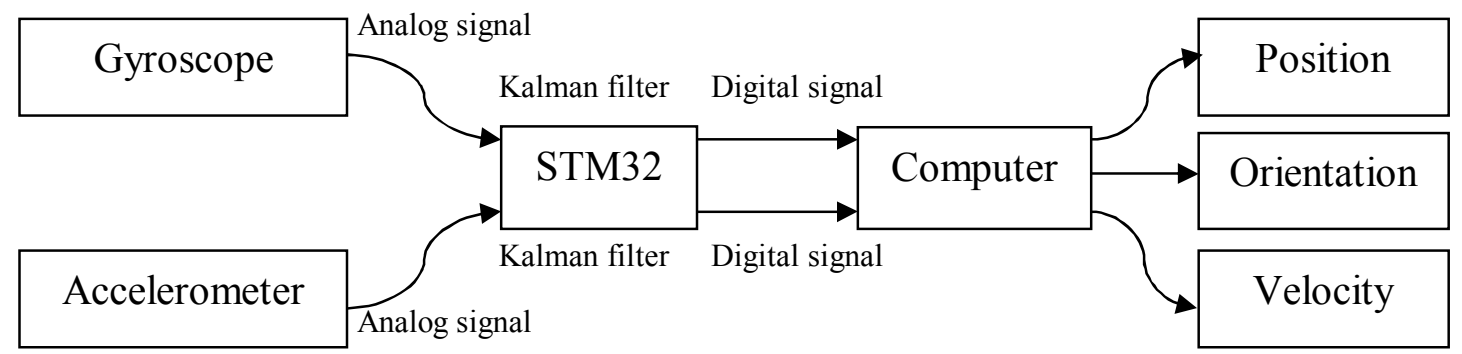

Fig. 1 Schematic of fixed-fixed microswitch

To solve above two problems, we design a data acquisition device based on STM32 for inertial navigation system. This data acquisition devices has analog to digital conversion and Kalman filtering function, when the measured value of the gyroscope and accelerometer through its processing into the computer later, the computer can calculate the accurate navigation information. The New design of inertial navigation system framework as shown in Fig. 1. 


\section{Kalman filter}

Kalman filtering is an optimal state estimation process applied to a dynamic system that involves random perturbations[3]. More precisely, the Kalman filter gives a linear, unbiased, and minimum error variance recursive algorithm to optimally estimate the unknown state of a dynamic system from noisy data taken at discrete real-time[4]. Combining with the appeals (b), obviously, the Kalman filter is ideal for Inertial Navigation System[5].

As follows, we designed the kalman filtering algorithm for the measured value of the gyroscope and accelerometer :

$\hat{x}_{k / k}=\hat{x}_{k / k-1}+\boldsymbol{G}_{k}\left(\boldsymbol{y}_{k}-\boldsymbol{C} \hat{x}_{k / k-1}\right)$

$\hat{x}_{k / k-1}=\boldsymbol{A}_{k-1} \hat{\boldsymbol{x}}_{k-1 / k-1}$

$\boldsymbol{P}_{k, k-1}=\boldsymbol{A}_{k-1} \boldsymbol{P}_{k-1, k-1} \boldsymbol{A}_{k-1}^{T}+\boldsymbol{L}_{k-l} \boldsymbol{Q}_{k-1} \boldsymbol{L}_{k-1}^{T}$

$\boldsymbol{G}_{k}=\boldsymbol{P}_{k, k-l} \boldsymbol{C}_{k}^{T}\left(\boldsymbol{C}_{k} \boldsymbol{P}_{k, k-1} \boldsymbol{C}_{k}^{T}+\boldsymbol{R}_{k}\right)^{-1}$

$\boldsymbol{P}_{k, k}=\left(\boldsymbol{I}-\boldsymbol{G}_{k} \boldsymbol{C}_{k}^{T}\right) \boldsymbol{P}_{k, k-1}$

$\hat{x}_{k / k}$ : The output value of filter, that is, the optimal estimate.

$\hat{x}_{k-1 / k-1}$ : The actual measured value, that is, he measured value of the gyroscope and accelerometer.

$\boldsymbol{y}_{k}$ : The predictive value.

$\boldsymbol{G}_{k}$ : Kalman Gain.

$\boldsymbol{A}_{k-1}$ : The system state transition matrix.

$\boldsymbol{C}_{k}$ : The system prediction matrix.

$\boldsymbol{P}_{k}$ : The variance of predictive value.

$\boldsymbol{R}_{k}$ : The variance of measured values.

According to the above algorithm, we design the STM32 kalman filtering program, its frame as shown in Fig. 2:

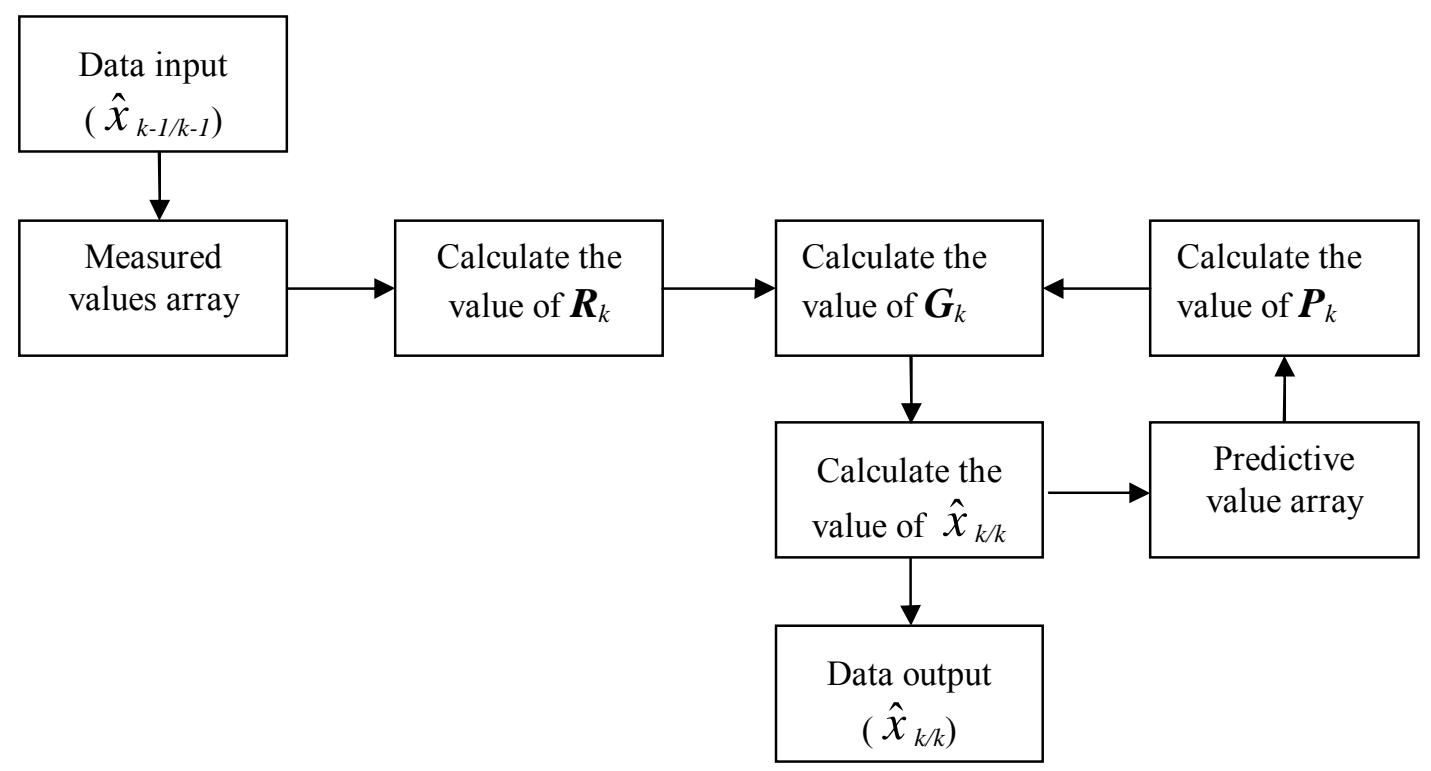

Fig. 2 The STM32 kalman filtering program frame

In the figure above,the measured values array and the predictive value array both contains 100 values( The capacity of the two arrays can be modified according to the situation), and constantly update their values.

\section{Architecture of data acquisition device}

STM32 is a family of 32-bit microcontroller integrated circuits by STMicroelectronics. Here we use STM32F407VGT6 as control chip, it is the first group of STM32 microcontrollers -based on the ARM 
architecture M4F core. STM32F407VGT6's core at a maximum clock rate of $168 \mathrm{MHz}$, and the capacity of the RAM is $192 \mathrm{~KB}$. Moreover, it has three 12-bit analog-to-digital converters(ADC) .

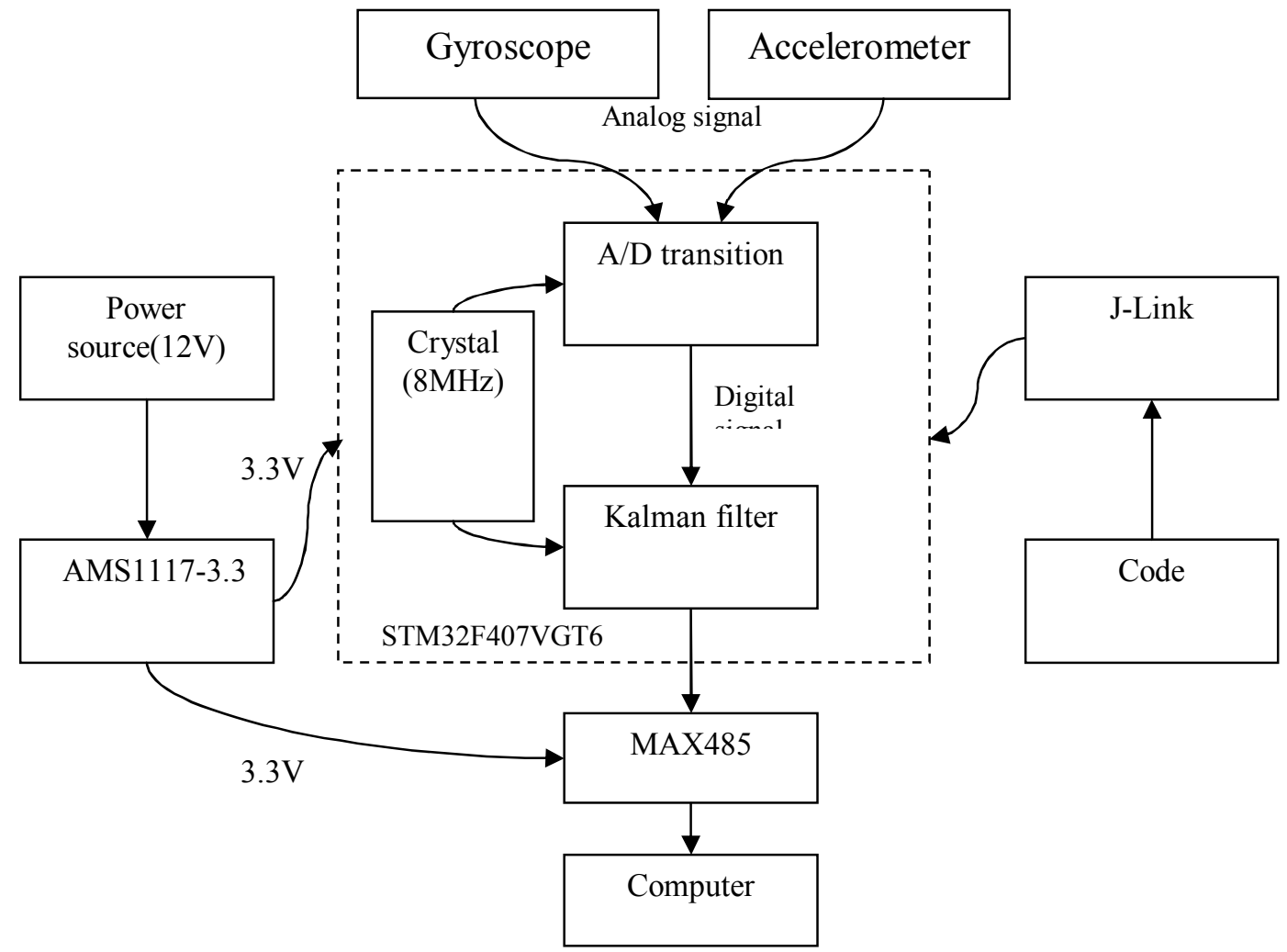

Fig. 3 The data acquisition equipment functional diagram

Fig. 3 shows the architecture of data acquisition device, here are some details:

a.The ADCs of STM32F407VGT6 is a successive approximation A/D converter. Their maximum sampling frequency is $36 \mathrm{MHz}$, and the sampling frequency can be set according to the actual situation.

b.MAX485 is a chip with RS485 interface, which uses half-duplex communication. MAX485 can achieve a maximum transmission rate of $2.5 \mathrm{Mbps}$, and has a strong ability to resist noise interference.Its maximum transmission distance can reach $3000 \mathrm{~m}$.

c. The power circuit is mainly composed of voltage stability chip AMS1117-3.3, it converts external voltage from $12 \mathrm{~V}$ to $3.3 \mathrm{~V}$, ensuring the normal work of data acquisition device.

d. The J-Link is used to download the code to STM32.

\section{Experiments}

Inclinometer is one of the members of the inertial navigation system. In order to analyze the performance of the data acquisition device based on STM32, we made one for a 2-Axis Digital Inclinometer and conducted experiments.

The experiment of the data acquisition device has been done and data acquisition comes from the MEMS accelerometers of 2-Axis Digital Inclinometer, which is installed on the bench of rotating machine.

The data acquisition have displayed as shown in Fig.4. In this figure, the abscissa is the measured angle value of the inclinometer, the ordinate is the actual value of the tilt angle. We can see from Fig. 4, the measured value substantially equal to the actual value.

More accurate relationship between actual and measured values as shown in Fig. 5. In this figure, the abscissa is the ordinate is the actual value of the tilt angle, the ordinate is the measurement error of the inclinometer. ( Measurement error $=$ The measured value - The actual value) We can see from Fig. 5 , the biggest measurement error of the inclinometer is $0.07^{\circ}$. Therefore, the inclinometer completely accords with the accuracy requirement of the 2-Axis Digital Inclinometer(Measurement error $\leqslant 0.1^{\circ}$ ). 


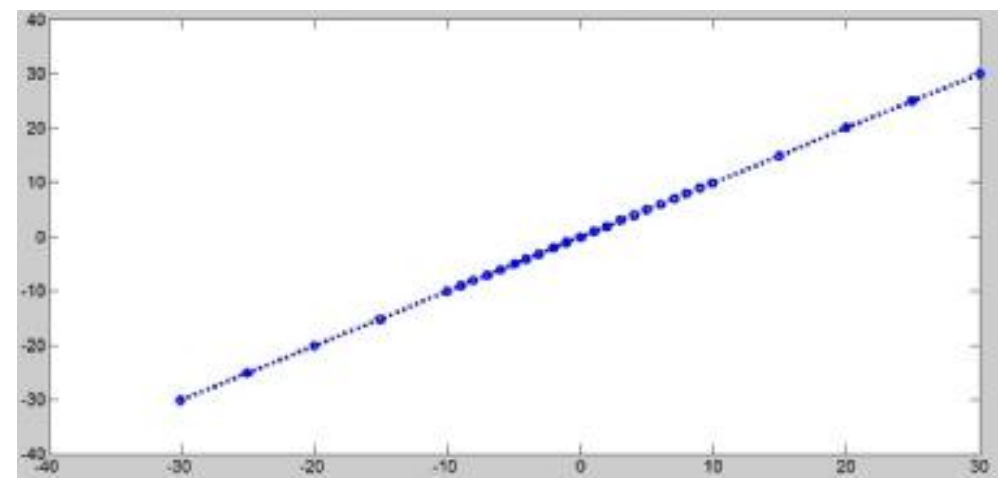

Fig. 4 The relationship between the measured value and the actual value

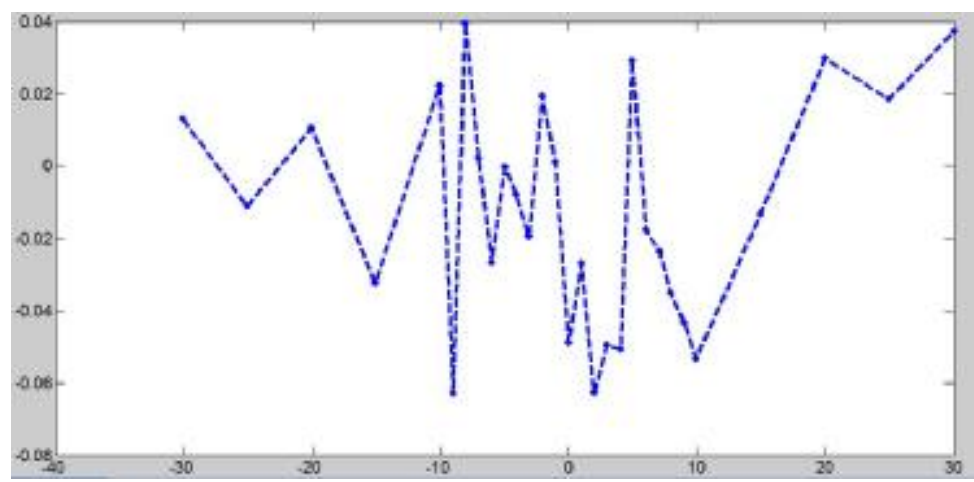

Fig.5 Measurement error

\section{Conclusions}

We design a new INS data acquisition device based on STM32, and introduces the design of the kalman filtering program. and the architecture of data acquisition device. After that, we use the data acquisition device in inclinometer. The experimental test shows that the data acquisition device accurately realized the function of analog-to-digital conversion and filtering.

\section{Acknowledgements}

Our works are supported by Natural Science Foundation of China (51407036) and Guangxi Science Foundation (2013GXNSFCA019019, 2014GXNSFBA118274) .

\section{References}

[1] Kuritsky M M, Goldstein M S, Greenwood I A, et al. Inertial navigation. Proceedings of the IEEE, 1983, 71: 1156-1176.

[2] Weston J L, Titteron D H. Modern inertial navigation technology and its application, Electronics and Communication Engineering Journal, 2000, 12 (2) : 49-64.

[3] Charles K, Chui, Chen G. Kalman Filtering With Real-Time Applications[M]. Springer, 2008-12-01.

[4] Mazzà Claudia. An optimized Kalman filter for the estimate of trunk orientation from inertial sensors data during treadmill walking. [J].Gait \& Posture,2011,35(1)

[5] M.E. Gharamti. Complex step-based low-rank extended Kalman filtering for state-parameter estimation in subsurface transport models. [J].Journal of Hydrology,2014,509 